JURE ZOVKO

\title{
Zwischen Moderne und Tradition
}

\author{
Schlegels Europa-Idee
}

Wenn man über einen Klassiker schreibt - und Friedrich Schlegel gilt als ein solcher - hat man stets zu gewärtigen, dass man in seinem Werk auf übersehene und nicht ausreichend gewertete Bereiche seines Schaffens stößt. Jacob Burckhardt hat entsprechend auf das unumgängliche Bedürfnis hingewiesen, Werke der klassischen Autoren behutsam und sorgfältig zu studieren, weil in ihnen immer mehr und anderes zu lesen ist, als die Interpreten bisher erschlossen haben:

Die Quellen aber, zumal solche, die von großen Männern herrühren, sind unerschöpflich, so dass jeder die tausendmal ausgebeuteten Bücher wieder lesen muss, weil sie jedem Leser und jedem Jahrhundert ein besonderes Antlitz weisen [...]. Es kann sein, daß im Thukydides z. B. eine Tatsache ersten Ranges liegt, die erst in hundert Jahren jemand bemerken wird. ${ }^{1}$

Friedrich Schlegel hat I 50 Jahre zuvor, im oft zitierten Lyceumsfragment Nr. 20, in ähnlicher Weise angemerkt: „Eine klassische Schrift muß nie ganz verstanden werden können. Aber die, welche gebildet sind und sich bilden, müssen immer mehr draus lernen wollen“. ${ }^{2}$ Bildung steht im Vordergrund des Schlegelschen Denkens und enthält als solche den Status der Platonischen ,ontos onta'. Da Bildung keine abstrakte Gegebenheit ist, sondern das Medium der hermeneutischen Reflexion darstellt, versucht Schlegel vor allem die Bedeutsamkeit der Tradition für die Bildung hervorzuheben.

Am Beispiel der Analyse altgriechischer Poesie hat Schlegel veranschaulicht, wie im hermeneutisch-kritischen Studium die Erforschung der antiken Literatur unternommen wird, um das

I Jacob Burckhardt: Weltgeschichtliche Betrachtungen. Stuttgart I929, S. I 5 f.

2 Friedrich Schlegel wird zitiert nach der Kritischen Friedrich-Schlegel-Ausgabe (künftig zitiert als KFSA). Hg. v. Ernst Behler unter Mitw. v. Jean-Jacques Anstett u. Hans Eichner. Paderborn I 958 ff; hier: KFSA 2, S. I 49. 
140 Verständnis und die Charakterisierung der modernen Dichtung zu begünstigen bzw. kreative Ideen für die weitere Kunstentwicklung zu erhalten. Gerade im Studium der literarischen Überlieferung, die aus der weiten Vergangenheit in die lebendige Gegenwart des Gesprächs hineinzuholen ist, erkennt er ihre strukturelle Differenz gegenüber der modernen Literatur: Die antike Poesie ist nach dem Prinzip der reinen Schönheit strukturiert, ${ }^{3}$ die moderne Literatur dagegen hat das Interessante, Individuelle und Charakteristische zum Prinzip des künstlerischen Schaffens gemacht. ${ }^{4}$ In diesem $\mathrm{Zu}$ sammenhang hat Emil Staiger vom Streit zwischen Schiller und Schlegel hinsichtlich der Kriterien, wie Kunstwerke konzipiert werden sollten, gesprochen, wobei nach Staiger bedauerlicherweise Friedrich Schlegel den Sieg errungen habe. 5 Trotz der innovativen Kriterien, mit denen das Klassische in der Kunst anscheinend desavouiert wird, plädiert Schlegel für die behutsame Interpretation und intensive Lektüre der klassischen Werke, weil sich diese als kompetente Gesprächspartner erweisen, die imstande sind - wie Schlegel in seinem Lessing-Aufsatz behauptet -, das Selbstdenken zu erregen. Seine außergewöhnliche Virtuosität auf dem Gebiet der ,kritischen' Interpretation hat Schlegel in seinen zahlreichen Charakteristiken und Kritiken über griechische Dichtung, über Boccaccio, Shakespeare, Herder, Lessing, Goethe, Condorcet, Kant und Jacobi, über indische Poesie und Religion sowie über die gotische Baukunst und die Gemälde der Renaissance nachgewiesen. Mit seiner Charakteristik des Plato hat Schlegel paradigmatisch gezeigt, wie die Kunst der Interpretation auf das Kunstwerk eines philosophischen Klassikers appliziert werden sollte. ${ }^{6}$

Das Konzept einer verantwortlichen Interpretation von klassischen Texten hat Schlegel aus der traditionellen ars critica abgeleitet, einer Hilfsdisziplin der klassischen Philologie, die es vorzugsweise mit der restitutio eines Textes zu tun hat. Seit etwa I 797 versucht er, eine ,hermeneutische Kritik' als Theorie vom Verstehen und

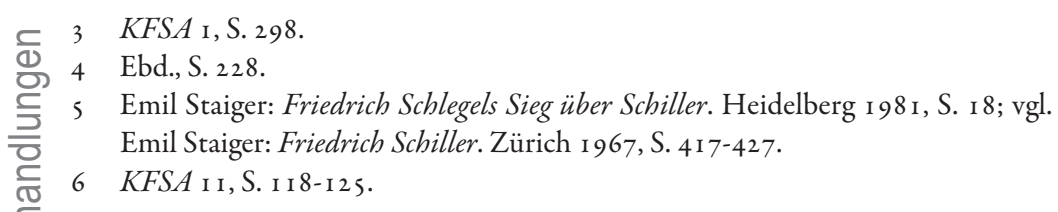


Auslegen der Texte auszuarbeiten, deren primäre Aufgabe es ist, die intersubjektive Dimension des Sinnes zu ermitteln und einen symproduktiven dialogischen Gedankenaustausch mit dem Kunstwerk zustande zu bringen. In diesem Zusammenhang hat Ernst Behler durchaus Recht, wenn er behauptet, dass die Theorie des Verstehens das Motto und der einheitliche Faden in Schlegels vielschichtigem Opus bleibt: „Daß Schlegels gesamtes Lebenswerk vom Thema des Verstehens geleitet ist, scheint ohne Frage zu sein "7. Die Lektüre verstand Schlegel in seiner kritischen Epoche als Kunst des ,symphilosophierenden' Gespräches mit dem Text, in welchem der kreative Schaffensprozess fortgesetzt wird, weil der „synthetische Schriftsteller" schon bei der Abfassung seines Werkes mit seinem zukünftigen Leser ,in das heilige Verhältnis der innigsten Symphilosophie oder Sympoesie " tritt. ${ }^{8}$ Entsprechend ist es die verantwortungsvolle Aufgabe des Interpreten, den angebotenen Diskurs weiterzuführen. Schlegels Behauptung, dass „Texte für das Studium oder die Symphilosophie da stehen," "verweist auf den bivalenten Charakter der Interpretation: Die zu verstehenden Werke sind einerseits Objekte potentieller wissenschaftlicher Analyse, wie dies in der philologischen oder historischen Behandlung eines alten Textes der Fall ist, und andererseits erweisen sich diese Werke als aktive Dialogpartner, mit denen man ins Gespräch kommt und deren veritativen Charakter man erforscht. Es wird gleichfalls hervorgehoben, dass nicht alle Werke des interpretierenden Symphilosophierens wert sind, sondern lediglich diejenigen, „die à la bauteur sind“. ${ }^{10}$ In der Auseinandersetzung mit dem starken Gesprächspartner bereichert der Interpret seine Kenntnisse, erweitert seinen Horizont, schärft seine Urteilskraft und gibt Hinweise zur innovativen und kreativen Kunstentwicklung. Das kritisch-hermeneutische Lesen stellt sich mithin als ein heuristischer Prozess der dynamischen Wechselwirkung heraus, in welchem der

7 Ernst Behler: „Friedrich Schlegels Theorie des Verstehens: Hermeneutik oder Dekonstruktion“. In: Ders./Jochen Hörisch (Hg.): Die Aktualität der Frühromantik. Paderborn 1987, S. 141-160, hier: S. I45.

$8 K F S A$ 2, S. I61, Nr. I I 2

9 Ebd., S. 177, Nr. 82

Io Ebd., S. 2 10, Nr. 264. 
142 Interpret offen bleibt für das, was das Werk zu sagen hat, während das Werk im Verstehen seine neue Belebung erfährt bzw. im Bewusstsein des Lesers neu konstruiert wird.

In seiner Rezension der vier ersten Bände von Niethammers Philosophischem Journal thematisiert Schlegel die Grundlinien seiner hermeneutischen Kritik. Mit der provozierenden Frage: „Wie sollte es ein Wissenschaftsurteil geben, wo es noch keine Wissenschaft gibt?" "II, versucht er eine hermeneutische Notwendigkeit zum Ausdruck zu bringen, dass nämlich die Erforschung und Auswertung der philosophischen Überlieferung Kompetenz und kultivierte Urteilskraft des Interpreten voraussetzt. Beachtenswert ist in diesem Zusammenhang auch ein Fragment über die Relevanz des kompetenten Verstehens und der kritischen Würdigung:

Man soll über die Kunst philosophiren, denn man soll über alles philosophiren; nur muß man schon etwas von der Kunst wissen. - Freilich wird alles was man von der Kunst erfahren hat, erst durch $\phi \sigma[$ Philosophie $]$ zum Wissen. ${ }^{12}$

Man muss nach Schlegels Ansicht immer schon vieles verstanden haben, bevor man sein Kunsturteil fällt, erst nach langer hermeneutischer Erfahrung erreicht der hermeneutische Kritiker jenes Stadium, in dem er imstande ist, "das Verstehen zu verstehen“, wie es Schlegel im i 80 i erschienenen Abschluss des Lessing-Aufsatzes formuliert hat. ${ }^{13}$ Erst dadurch kommt man zu der Einsicht, dass Verstehen keine abstrakte, sondern eine integrative Tätigkeit ist, deren Zweck die Erweiterung und Vertiefung der menschlichen Bildung ist. Durch die Bildung, heißt es in den Ideen, „wird der Mensch, der es ganz ist, überall menschlich und von Menschheit durchdrungen " ${ }^{14}$ Die Bildung erweist sich als das Verbleibende und Zuverlässige, worauf man sich stützen kann. Sie ist das Ein-

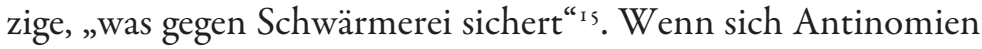

I I $K F S A 8$, S. 30.

I2 $K F S A$ i6, S. Iо I, Nr. 193.

I3 KFSA 2, S. 4 I 2.

14 Ebd., S. 262, Nr. 65. Vgl. auch ebd., S. 266, Nr. 98 : „Denke Dir ein Endliches ins Unendliche gebildet, so denkst du einen Menschen".

is KFSA i $8, S$. 5 i 8 , Nr. 13. 
„zwischen mehren Theilen d[er] menschl.[ichen] Bildung“ erge-

ben, wird unter ihnen nicht notgedrungen eine harmonische Versöhnung angestrebt, sondern es wird vielmehr darin die bereichernde Möglichkeit zu einer dialogischen „Wechselbestimmung " gesehen. ${ }^{16}$ Schlegels Diktum aus dem Nachlass, „kp[Kritik] ist das allgemeine Bildungsmittel ${ }^{{ }^{117}}$, wird im Gespräch über die Poesie ausführlicher entfaltet und ausgearbeitet. Allein die „hohe Wissenschaft echter Kritik“ vermag einen bildungsinteressierten Menschen zu lehren,

wie er sich selbst bilden muß in sich selbst, und vor allem soll sie ihn lehren, auch jede andre selbständige Gestalt der Poesie in ihrer klassischen Kraft und Fülle zu fassen, daß die Blüte und der Kern fremder Geister Nahrung und Same werde für seine eigne Fantasie. ${ }^{18}$

Klassische Werke studiert man folglich nicht bloß um der Vertiefung und Vervollkommnung der eigenen Bildung willen. Es geht in dieser Form des Studiums vielmehr um Inspiration für die eigene künstlerische Tätigkeit und Kreativität.

Der verstehende Prozess fungiert streng betrachtet poietisch in einem ausgezeichneten Sinne des Wortes, er wird als eine progressive, ,antithetische Synthesis “vollzogen, die Schlegel paradoxerweise als eine fortgehende „Kette der ungeheuersten Revoluzionen“ charakterisiert. ${ }^{19}$ Im Bildungsprozess werden durch die kritische Reflexion über die klassischen Werke der Tradition die inneren Potentiale des Interpreten vertieft und die Kultivierung der eigenen Person bzw. der eigenen Urteilsfähigkeit wird ermöglicht. Dasselbe gilt freilich für die geschichtliche Welt, die immer nach- und fortgebildet, aber nie vollendet wird. Die Konzeption einer unvollendeten Welt erweist sich für Schlegel als conditio sine qua non für die ,humane Selbstbehauptung' des Menschen der Moderne. Im Unterschied zu Kant, der noch die creatio continua als Grundbe-

I6 Vgl. KFSA I 8, S. 301, Nr. I 286.

17 Ebd., S. 374, Nr. 646.

I $8 K F S A$ 2, S. 284.

19 $K F S A$ I 8, S. 82 f., Nr. 637. 
144 stimmung und primäre Aufgabe der Natur betrachtet, geht Schlegel in seiner Jenaer Vorlesung über Transcendentalphilosophie davon aus, „[d]aß die Welt noch unvollendet ist ${ }^{“ 20}$. Daraus folgert er:

$[S]$ o ist unsere Bestimmung wohl, an der Vollendung derselben mitzuarbeiten. [...] Wäre die Welt vollendet, so gäbe es dann nur ein Wissen derselben, aber kein Handeln. ${ }^{21}$

Die Transcendentalphilosophie arbeitet mit einem durchaus kreativen Konzept der vita activa: „Der Mensch dichtet gleichsam die Welt, nur weiß er es nicht gleich. ${ }{ }^{2}$

Die Ansicht, dass Schlegel ein Kohärenzmodell der Wahrheit vertritt, wonach unterschiedliche, sich wechselseitig stützende Meinungen zu einem in sich stimmigen Ganzen vereinheitlicht werden ${ }^{23}$, lässt sich $\mathrm{m}$. E. lediglich vor dem hermeneutischen Hintergrund und Standpunkt seines Denkens rechtfertigen, namentlich durch den Gedanken des hermeneutischen Zirkels und dessen Anwendung in der hermeneutischen Praxis des Verstehens und Auslegens von Texten. Der hermeneutisch-kritische Vorgang, wie Schlegel ihn in den Heften Zur Philologie konzipiert hat, fungiert

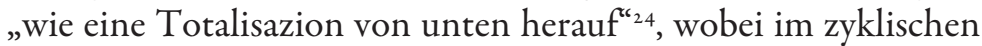
Verfahren des Lesens die Bedeutung des Einzelnen im Kontext des Ganzen aufgefasst und der Sinngehalt des Ganzen durch das Verständnis der Einzelteile mitkonstituiert wird. Schlegels Konzept einer Philosophie der Philologie impliziert Liebe zum und Leidenschaft für das Wort; das Verstehen der Wortbedeutung erfordert wiederum Kenntnis und Verständnis des breiteren Kontextes der Sprache, des Werkes und der gesamten geistigen Bildung des Au-

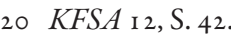

2 I Ebd.

22 Ebd., S. Io5

23 Vgl. Manfred Frank: „Unendliche Annäherung“. Die Anfänge derphilosophischen Frühromantik. 2. Aufl. Frankfurt a. M. 1997; Ders.: „Wechselgrundsatz. Friedrich Schlegels philosophischer Ausgangspunkt“. In: Zeitschrift für philosophische Forschung 50 (1996), S. 26-50, hier: S. 49 f.; Birgit Rehme-Iffert: Skepsis und Enthusiasmus. Friedrich Schlegels philosophischer Grundgedanke zwischen I796 und I805. Würzburg 200 I, S. 59 f., S. 86 ff.

24 KFSA I 6, S. 68, Nr. 84. 
tors. „Die erste Bedingung alles Verständnisses“, heißt es in Les-

sings Gedanken und Meinungen, „und also auch das Verständnis eines Kunstwerks, ist die Anschauung des Ganzen“"25. Der „Gang und Gliederbau " ${ }^{\text {"26}}$, die Eigentümlichkeiten seiner Bestandteile des Ganzen sollen dabei genau erfasst und nachkonstruiert werden. ${ }^{27}$ Schlegel warnt im Abschluss des Lessing-Aufsatzes vor der einseitigen Interpretation der einzelnen Textteile außerhalb ihres Kontextes:

Daß man im Kunstwerke nicht bloß die schönen Stellen empfinden, sondern den Eindruck des Ganzen fassen müsse; dieser Satz wird nun bald trivial sein, und unter die Glaubensartikel gehören. Weiter noch gehen die Philosophen, und fodern, ja versuchen, sich selbst und andre im ganzen zu verstehen, mag der Autor auch dieses Ganze, den gemeinsamen Geist in einen noch so geistlosen Buchstaben gehüllt, und in eine sehr komplizierte Reihe vieler, vielleicht etwas konfuser Schriften zerstreut haben. ${ }^{28}$

Knapper gefasst: Der entsprechende „Glaubensartikel“ der hermeneutischen Kritik lautet, „daß man das Werk nur im System aller Werke des Künstlers ganz verstehe “29. Auch wenn jemand, wie Schlegel selbst, die Tendenz hat, sich vorwiegend fragmentarisch und gleichsam unsystematisch auszudrücken, bleibt die Aufgabe des Interpreten zu erschließen, ob sich sein Schaffen in einen „organische[n] Zusammenhang " ${ }^{30}$ integrieren lässt. Ferner obliegt es dem Interpreten, den Autor bzw. sein Werk im Kontext der gesamten kulturellen und geisteswissenschaftlichen Errungenschaften $\mathrm{zu}$ prüfen bzw. zu erfragen, welche der vier kritischen Kategorien auf den jeweils bestimmten Text applizierbar sind. ${ }^{31}$

$25 \mathrm{KFSA}_{3}, \mathrm{~S}_{5} 6$

26 Ebd., S. 60.

27 Ebd., S. 58.

28 KFSA 2, S. 4 Io.

29 Ebd.

30 Ebd.

3 I Vgl. KFSA 2, S. 4 I 2 f.: ,Wenn ihr versuchen wollt, Autoren oder Werke zu verstehen, d. h. sie in Beziehung auf jenen großen Organismus aller Kunst und Wissenschaft genetisch zu konstruieren; so werdet Ihr bemerken, daß es vier 
146 Der mit der Bildung gleichgesetzte hermeneutisch-kritische Prozess des Verstehens hat die Form eines zyklischen Verfahrens und vollzieht sich als eine bis ins Unendliche fortgehende, progressive „antithetische Synthesis. “ ${ }_{32}$ Das progressive Fortbilden der tradierten Sinneinheiten gleicht einer offenen Spirale, wobei sich das vorläufig Verstandene und Interpretierte ipso facto als das Revisionsbedürftige erweist. Hermeneutische Kritik als „,eine universale philosophische Kunst" stellt sich demzufolge als eine mit der „, $\mu \phi[$ Symphilosophie] " gleichgesetzte,$\phi \phi[$ Philosophie der Philosophie]" heraus ${ }^{33}$, deren Zweck nicht nur im zuverlässigen Verstehen des tradierten Interpretandum liegt, sondern auch in der Neuorientierung der kreativen Tätigkeit des menschlichen Geistes. In einem Fragment, das er in die unter dem Namen Novalis im Athenaeum veröffentlichte Sammlung Blüthenstaub eingefügt hat, fragt Schlegel: „[I]st das Leben eines denkenden Menschen wohl etwas andres als eine stete innere Symphilosophie?" ${ }^{34}$ Die Lebensformen, mit denen unser Individuum in seinem Existenzvollzug ,sym-philosophiert', sind die geschichtlichen Objektivationen und Artikulationen des menschlichen Geistes. Schlegel ist vor allem an der Kontinuität der europäischen Kultur in ihren Transformationen interessiert, da sich für ihn das Leben der europäischen Völker in der literarischen Welt geschichtlich und kulturell reflektiert.

Bereits in Schlegels Abschluss des Lessing-Aufsatzes lässt sich eine Wende diagnostizieren, die Matthias Schöning als Ironieverzicht gekennzeichnet hat ${ }^{35}$ : Schlegel kündigt dort an, sich künftig auf die „Geschichte der Dichtkunst" und die „Kritik der Philosophie“ zu konzentrieren. ${ }^{36}$ Mit dieser Intention hängt auch seine Gründung der Zeitschrift Europa - im Anschluss an den Umzug nach Paris

\footnotetext{
Kategorien gibt, in die sich alles scheidet, was Ihr bei einer solchen Konstruktion Charakteristisches in dem Phänomen der Kunstwelt findet; vier Begriffe, unter die sich das alles fügt: Form und Gehalt, Absicht und Tendenz. Aber nicht alle diese Kategorien sind auf jedes Werk, auf jeden Autor anwendbar".

$32 K F S A$ i $8, S .82$, Nr. 637.

ᄃ 33 Ebd., S. 3 I 4 , Nr. 1454.

34 Athenaeum I (1798), S. 75.

35 Matthias Schöning: Ironieverzicht. Friedrich Schlegels theoretische Konzepte zwischen "Athenäum" und "Philosophie des Lebens". Paderborn u. a. 2002.

36 KFSA 2, S. 409 
im Spätherbst I 802 - zusammen. Hier macht er - im program-

matischen Essay Reise nach Frankreich - auf die einheitlichen Grundzüge der europäischen Geistesgeschichte aufmerksam; insbesondere auch für die Zeit, in der „Europa, moralisch und politisch genommen, schon völlig zerstört und untergegangen war" ${ }^{\text {" }}{ }^{77}$ In seinen ebenfalls in der Europa erschienenen Beiträgen zur Geschichte der modernen Poesie begrüßt Schlegel emphatisch die Tatsache, dass einige vortreffliche deutsche Dichter sich bemüht haben,

die Schönheiten der italiänischen und der spanischen Poesie auf einheimischen Boden zu verpflanzen, da der frische Blütenreiz und die kunstreiche Zierde derselben recht eigentlich dazu gemacht scheinen, den nordischen Ernst altdeutscher Dichtkunst zu schmücken und zu erheitern. ${ }^{38}$

Im Louvre erkannte Schlegel die herausragende Relevanz der Malerei für die europäische Geistesgeschichte: Die Malerei erweist sich als spezifisch christliche Kunst, die die klassische Plastik abgelöst und sich als universelle Kunstgattung der Romantik etabliert hat. Diese Entdeckung ist m. E. ein Verdienst Friedrich Schlegels und nicht etwa Hegels, wie üblicherweise in der Geschichte der Ästhetik dargestellt. ${ }^{39}$ Dementsprechend schreibt Schlegel in seinem Europa-Essay Nachtrag italiänischer Gemälde:

Die Poesie der alten Maler war teils die Religion, wie beim Perugino, Fra Bartolomeo und vielen andern Alten; teils Philosophie, wie beim tiefsinnigen Leonardo, oder aber beides, wie in dem unergründlichen Dürer. ${ }^{40}$

Schlegels Wende zum vielfältigen Reichtum der europäischen Kultur war ein Schritt des verantwortlichen Kunsttheoretikers und Kritikers, der sich als damit als anerkannter Kulturforscher

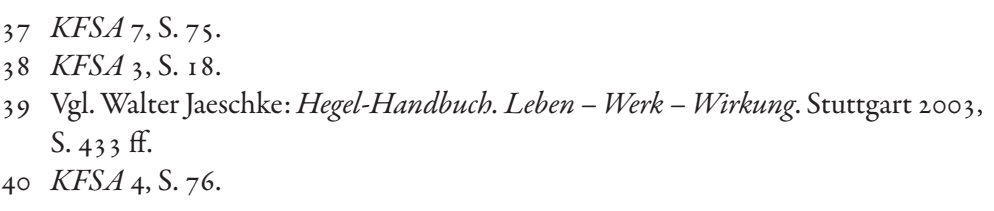


148 in fast allen Bereichen der Geisteswissenschaften erweisen sollte mit dem eindeutigen Zweck, die Fülle und den Reichtum der europäischen Kultur zu recherchieren und ihre Relevanz für die Epoche der Moderne zu ergründen. Seit seinem I 808 erfolgten Übertritt zum Katholizismus findet bei Schlegel eine eindeutige Interessenverschiebung statt: Er versucht seit diesem Zeitpunkt, sich in die bestehende sittliche Lebenswelt einzuleben und aus solcher Faktizität heraus eine eigene Lebensphilosophie auszuarbeiten. Schlegels Intention ist ferner, die miserable Situation der Abstraktheit der mechanistischen Welt- und Menschenkonzeption aufzuheben, in der "der Mensch selbst fast zur Maschine geworden“41 ist. Im Brennpunkt seines Interesses steht nun auch ein neues, anspruchsvolles Vorhaben, das in der Widmung der I 8 I 2 in Wien gehaltenen Vorlesungen zur Geschichte der alten und neuen Literatur an den Fürsten Metternich umrissen wird: Nun geht es Schlegel darum „der großen Kluft“ entgegenzuwirken, „welche immer noch die literarische Welt und das intellektuelle Leben des Menschen von der praktischen Wirklichkeit trennt" ${ }^{\text {42 }}$. Die Hinwendung zur kulturellen Lebenswelt der europäischen Geistesgeschichte bedeutet zugleich eine Distanznahme von den Grundlinien des Deutschen Idealismus. Während im ersten Band der Zeitschrift Europa "der Idealismus als die wesentliche Bedingung sine qua non, als Erhaltungsmittel und Grundlage unserer neuen Literatur "43 gilt, wird in den Kölner Vorlesungen diese bedeutende philosophische Formation der Moderne als System betrachtet, das den Menschen in „absolute Einsamkeit“ führt und daher unter allen philosophischen Systemen „,am meisten Furcht erregt“. ${ }^{44}$ Die Ohnmacht des einst gefeierten Individuums versucht Schlegel jetzt durch eine positive bzw. poetische Religion zu überwinden. Dies ließe sich in gewisser Hinsicht als Realisierung des romantischen Antriebs deuten, eine neue Mythologie aus der Tiefe des Geistes hervorzubringen. In der Reise nach Frankreich schreibt Schlegel:

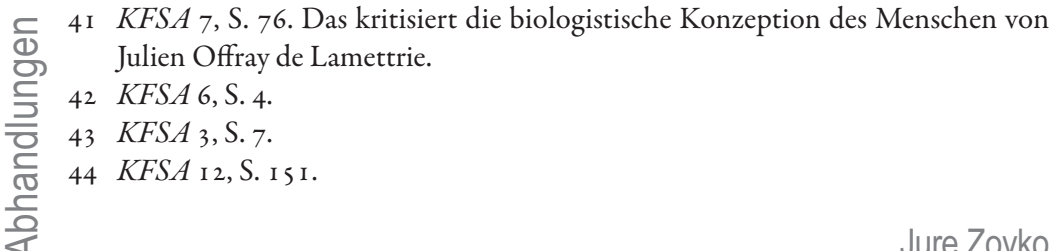


Zuerst ist es der katholischen Religion allerdings bis auf einen gewissen Grad gelungen, den künstlerischen Glanz und Reiz, die poetische Mannigfaltigkeit und Schönheit der griechischen Mythologie und Gebräuche sich zu eigen zu machen, und wieder einzuführen, so weit das bei der gänzlichen Verschiedenheit und Einseitigkeit der Prinzipien möglich war. ${ }^{45}$

Im Blickpunkt der Explikation der Frage nach dem Verhältnis von Kunst und Leben steht nicht mehr die Entgegensetzung von Antike und Moderne, sondern die Vereinigung beider, wie aus dem I 823 publizierten Abschluss der zweiten Fassung des Gesprächs über die Poesie ersichtlich ist. ${ }^{46}$ In der Reise nach Frankreich wird die noch im Athenaeum proklamierte „europäische Trennung des Klassischen und des Romantischen“ als „eigentlich unnatürlich und durchaus verwerflich" gekennzeichnet; ${ }^{47}$ es bleibt Schlegels Grundfrage, wie in der Moderne der Geist des Altertums kritisch anzueignen ist. Er versucht nun, die kulturellen und geistigen Wurzeln der Moderne in der griechischen Tradition zu erblicken und von da aus der Kontinuität der europäischen Kultur in ihrer Pluralität nachzugehen. Ein Jahrhundert später folgt ihm u. a. der britische Dichter und Essayist T. S. Elliot mit großer Resonanz nach. 